\title{
Screening for lupus anticoagulant and anticardiolipin antibodies in women with fetal loss
}

\author{
M D Creagh, R G Malia, S M Cooper, A R Smith, S L B Duncan, M Greaves
}

\begin{abstract}
Sixty six women with first or second trimester fetal loss were investigated for the presence of lupus anticoagulant by routine coagulation tests and the dilute Russell's viper venom time with a platelet neutralisation procedure, and for raised anticardiolipin antibodies by an enzyme linked immunosorbent assay. Of 35 women with recurrent fetal loss, seven were positive for lupus anticoagulant and six had increased IgG anticardiolipin antibodies, while of 31 women with only one or two episodes of fetal loss, one had lupus anticoagulant and none increased IgG anticardiolipin antibodies. These findings were significantly different. There was no difference in the incidence of increased IgM anticardiolipin antibodies between the two groups (three and two cases, respectively). A further 11 women with intrauterine death in the third trimester were studied and lupus anticoagulant and raised $\operatorname{Ig} M$ anticardiolipin antibodies were found in one case. No woman was known to have systemic lupus erythematosus.
\end{abstract}

It is concluded that lupus anticoagulant and increased IgG anticardiolipin antibodies are independently associated with recurrent first and second trimester fetal loss and that such cases should be investigated, even in the presence of otherwise good health, by a comprehensive methodological approach.

University

Department of

Haematology, Royal

Hallamshire Hospital,

Glossop Road,

Sheffield S10 2JF

M D Creagh

R G Malia

$S$ M Cooper

$M$ Greaves

University

Department of

Obstetrics and

Gynaecology, Jessop

Hospital for Women,

Sheffield

A R Smith

S L B Duncan

Correspondence to:

Dr M D Creagh

Accepted for publication

25 July 1990
About $15 \%$ of all recognised pregnancies terminate in spontaneous fetal loss, mostly in the first or second trimesters. Recurrent fetal loss-that is, three or more episodes-is less common, probably affecting about $1 \%$ of women who become pregnant, but the successful outcome of a further pregnancy is less likely and hence investigation for a potential cause and appropriate treatment are priorities. In such cases the abnormalities most commonly observed include anomalies of the uterine body, endometrial infections, cervical incompetence, hormonal dysfunction and chromosomal aberrations, but in most affected women none of these factors seems to be operative. Recent interest has centred on a possible association between the presence of the lupus anticoagulant and recurrent fetal loss, as initially noted in a woman with sys- temic lupus erythematosus (SLE) by Nilsson. ${ }^{1}$ Studies, particularly in relation to miscarriage during first or second trimesters, have suggested an association with lupus anticoagulant and raised anticardiolipin antibodies, but the methods and conclusions are variable. ${ }^{2-4}$ Of such cases, some $50 \%$ have been in women with SLE.

Lupus anticoagulants are a heterogeneous group of acquired antiphospholipid antibodies and only recently have methods for the identification of lupus anticoagulant and quantitation of anticardiolipin antibodies been standardised, thus allowing low titre antiphospholipid antibodies to be detected reliably. Furthermore, several previous studies in failed pregnancy have relied on only one type of test for the detection of antiphospholipid antibodies. We therefore used the dilute Russell's viper venom time (DRVVT), with a platelet neutralisation procedure (PNP), in addition to routine coagulation tests and in combination with a standardised enzyme linked immunosorbent assay (ELISA) for IgG and IgM anticardiolipin antibody, to determine the true incidence of antiphospholipid antibodies in association with first and second trimester fetal loss and third trimester intrauterine death. We also studied women with normal pregnancies and those who were not pregnant. Altogether, 77 patients and 43 controls were studied.

\section{Methods}

From April 1986 to October 198983 women under the care of the University Department of Obstetrics and Gynaecology, Jessop Hospital for Women, Sheffield, were investigated, after a spontaneous fetal loss, as part of a clinical programme. Testing was usually performed within seven days of a clinical event, less frequently in the Pre-Pregnancy Clinic, or during pregnancy. Six women were excluded from analysis because in three pathology was identified that would account for loss of their pregnancy (cases of cervical incompetence, a balanced chromosomal translocation, and a twin-twin transfusion), and in a further three cases the records were incomplete. The largest group studied comprised 66 women who had sustained fetal loss in the first or second trimesters, as diagnosed by a gynaecologist and where possible confirmed by histology. A further 11 women who had been screened for idiopathic intrauterine death during the third trimester were included to compare their 
results with those of women with first or second trimester fetal loss, or both. No woman was known to have SLE.

Thirty healthy non-pregnant laboratory staff were recruited and samples obtained from 13 healthy pregnant women at the time of routine venepuncture in the antenatal clinic. Normal ranges were established for the DRVVT and anticardiolipin assays, there being no significant difference in the results of the two control groups.

Blood was collected, by syringe in $1 / 10$ th volume of $3 \cdot 2 \%(0 \cdot 109 \mathrm{M})$ trisodium citrate dihydrate, centrifuged at $2200 \times \mathrm{g}$ for 10 minutes, separated and tested. The prothrombin time, thrombin time, kaolin cephalin clotting time (KCCT) (Platelet substitute, Diagnostic Reagents Ltd, Thame, Oxford) and the fibrinogen concentration were estimated by standard methods ${ }^{5}$ and the platelet count was estimated using a Coulter Counter S Plus. Where the KCCT was prolonged a correction test was performed with a 1:1 mix of patient and normal plasma.

The DRVVT with a platelet neutralisation procedure was performed as previously described, ${ }^{6}$ with modifications. Russell's viper venom and platelet substitute (Diagnostic Reagents Ltd, Thame, Oxford) were used at optimal concentrations to give a DRVVT of 35-40 seconds with pooled fresh control plasma. A platelet neutralisation procedure was performed with washed, freeze-thawed normal platelets, similarly diluted. Results were expressed as a ratio of the patient time to that of controls, the normal range being the mean of the control groups $+/-2 S D(0 \cdot 9-1 \cdot 09)$. Patients were considered to have a lupus anticoagulant when the DRVVT ratio with platelet substitute was above the normal range, being decreased by the addition of excess phospholipid in the platelet neutralisation procedure to the normal range, or as in one case, by over $20 \%$.

IgG and IgM anticardiolipin antibodies were assayed by ELISA, as described by Harris et $a l^{7}$ using $10 \%$ adult bovine serum as a blocking agent and sample diluent. Results were initially expressed as either negative, weak, moderate or strong positive (weak positive being an optical density reading 3SD above the mean), and from the middle of 1986 in anticardiolipin units $(\mathrm{U} / \mathrm{ml})$, calculated against IgG and IgM standards supplied by $\mathrm{Dr}$ E N Harris, formerly of the Rayne Institute, London. Results greater than 3SD above the mean of the control groups were regarded as

Number and range of positive lupus anticoagulant (by DRVVT/PNP) and anticardiolipin antibody results in 77 women

\begin{tabular}{llll}
\hline Patient group & $\begin{array}{l}\text { Lupus anticoagulant } \\
\text { ratio }\end{array}$ & $\begin{array}{l}\text { IgG anticardiolipin } \\
\text { antibody } \\
U / m l\end{array}$ & $\begin{array}{l}\text { IgM anticardiolipin } \\
\text { antibody } \\
U / m l\end{array}$ \\
\hline $\begin{array}{c}\text { Fetal loss } \geqslant 3 \text { episodes } \\
(\mathrm{n}=35)\end{array}$ & $7(1 \cdot 1-2 \cdot 10)$ & $6(\mathrm{WP}-90 \cdot 0)$ & $3(5 \cdot 3-13 \cdot 4)$ \\
$\begin{array}{c}\text { Fetal loss }<3 \text { episodes } \\
(\mathrm{n}=31)\end{array}$ & $1(1 \cdot 19)$ & 0 & $2(5 \cdot 1-5 \cdot 5)$ \\
$\begin{array}{c}\text { Intrauterine death } \\
(\mathrm{n}=11)\end{array}$ & $1(1 \cdot 25)$ & 0 & $1(16 \cdot 3)$ \\
\hline WP $=$ weak positive. & & &
\end{tabular}

positive for anticardiolipin antibody-that is, for IgG greater than $8.0 \mathrm{U} / \mathrm{ml}$ and IgM greater than $5.0 \mathrm{U} / \mathrm{ml}$ ).

Statistical analysis of the positive DRVVT with platelet neutralisation procedure results was performed using Wilcoxon's rank sum test. Fischer's exact probability test was applied to the DRVVT with platelet neutralisation procedure and anticardiolipin antibody findings in the different patient groups.

\section{Results}

In view of previous reports on the incidence of lupus anticoagulant and anticardiolipin antibodies in women with recurrent fetal loss during the first and or second trimesters, we compared our findings in the women investigated for this $(n=35)$ with those women who had had one or two episodes $(n=31)$ and also with a group of women who had sustained an intrauterine death in the third trimester (table).

In six patients the KCCT was prolonged (range 46-67 seconds, normal range 32-45). All but two, however, corrected on mixing 1:1 with normal plasma, both of which were associated with a positive DRVVT with platelet neutralisation procedure. Minimal prolongation of the prothrombin time was found in two patients and thrombocytopenia in one patient (platelet count $138 \times 10^{9} / 1$ normal range $150-350 \times$ $\left.10^{9} / 1\right)$, there being no association with either lupus anticoagulant or increased anticardiolipin antibodies. The thrombin time and fibrinogen concentration were normal in all cases.

Among the women with first or second trimester fetal loss, or both, eight were considered to have lupus anticoagulant identified by the DRVVT (results ratio $1 \cdot 10-2 \cdot 10$, all correcting to the normal range with platelet neutralisation procedure, other than for a result of $2 \cdot 10$, which corrected to a ratio of $1 \cdot 30$ ), six had increased IgG anticardiolipin antibody titres (levels of weak positive, positive, $8 \cdot 4$, $11.9,29.7$ and $90.0 \mathrm{U} / \mathrm{ml}$ ), and five had increased $\operatorname{IgM}$ anticardiolipin antibody titres (levels of $5 \cdot 1,5.3,5.5,6.2$ and $13.4 \mathrm{U} / \mathrm{ml}$, respectively.)

\section{Discussion}

The findings of lupus anticoagulant and increased anticardiolipin antibodies in women with recurrent fetal loss have been shown previously, ${ }^{1-48-10}$ and in affected women further pregnancy has a failure rate of the order of $70 \%$. Various treatment regimens have been used including, alone or in combination, prednisolone, aspirin, azathioprine, heparin, plasmapheresis and immunoglobulin, with some success, although the results are conflictual. ${ }^{11}$ In view of the therapeutic possibilities it is important to confirm the validity of such observations, by standardised tests, and to define which patients should be screened. In the series reported, we therefore used a standardised ELISA for the determination of the anticardiolipin antibody, in combination with, we believe for the first time, identification 
and confirmation of the presence of lupus anticoagulant, by the DRVVT with the platelet neutralisation procedure, a sensitive and specific test comparable with the kaolin clotting time. ${ }^{12}$

Of the 77 women screened, 17 were found to be positive for lupus anticoagulant by the DRVVT with the platelet neutralisation procedure, an increased anticardiolipin antibody titre, or both. Among the 35 women who had had recurrent fetal loss, seven were positive for lupus anticoagulant, by the DRVVT with the platelet neutralisation procedure, six had increased IgG anticardiolipin antibodies, and two both; these 11 women had had 54 pregnancies in total, resulting in seven live births Of the 31 women who had had one or two episodes of fetal loss, one had a positive DRVVT with the platelet neutralisation procedure and none had increased IgG anticardiolipin antibody. These results were sig nificantly different for the two groups, there being an association between recurrent fetal loss and the finding of lupus anticoagulant $(p<0.05)$ and of increased IgG anticardiolipin antibodies $(p<0.02)$. There was no association between these findings, however, the coagulation based tests often being abnormal in the absence of a raised titre of IgG anticardiolipin antibody and vice versa. There was no significant difference in the incidence of $\operatorname{IgM}$ anticardiolipin antibodies between the two groups (three and two cases, respectively). There was a low incidence of findings in the women who had sustained an intrauterine death during the third trimester one woman being found to have had lupus anticoagulant in association with a raised IgM anticardiolipin antibody titre. The poor concordance between the results of the DRVVT with the platelet neutralisation procedure and the KCCT illustrates the lack of sensitivity and specificity if the KCCT alone were used as a screening test for lupus anticoagulant, and the necessity of an additional sensitive test. ${ }^{12}$

We believe that lupus anticoagulant and increased IgG anticardiolipin antibodies are independently associated with recurrent first or second trimester fetal loss, or both, there being a low incidence of these findings in women with one or two such episodes. The IgM anticardiolipin antibody titre does not discriminate between these two groups. Most of our patients with lupus anticoagulant did not have increased IgG anticardiolipin antibodies and would not have been identified without the use of a coagulation based assay, and this shows the necessity of using both means of investigation for antiphospholipid antibodies.

Petri et al reporting on a group of $\mathbf{4 4}$ women who habitually aborted, found an incidence of $9^{\circ}{ }^{\circ}$ lupus anticoagulant using the DRVVT, without a platelet neutralisation procedure, and $11 \%$ increased IgG, or IgM anticardiolipin antibodies by ELISA. ${ }^{9}$ These results were not significantly different from those of a control group. This contrasts with our findings, which in part may be due to the use of the platelet neutralisation procedure to increase the specificity of the DRVVT and of a standardised ELISA. Other series of women who recurrently miscarry, not known to have SLE, have identified lupus anticoagulant in $2 \%$ using the activated partial thromboplastin time and $48 \%$ by the addition of the dilute simplastin test. ${ }^{210}$ The finding of increased IgG anticardiolipin antibodies in seven of our group of 35 women who sustained recurrent fetal loss compares with $6.6 \%$ and $35.3 \%$ in other series. $^{34}$ These variations in the reported incidence of lupus anticoagulant and increased anticardiolipin antibodies are due in part to patient selection, pre-analytical variables, the heterogeneous nature of the lupus anticoagulant and the sensitivity of the various tests and confirm the importance of a standardised methodology.

In conclusion, we believe that lupus anticoagulant and increased anticardiolipin antibodies are underdiagnosed in women who repeatedly miscarry and that in view of the possibility of treatment it is vital that this group be investigated, even in the presence of otherwise good health, by a comprehensive methodological approach.

We are grateful to Ms L Westlake for assistance in the analysis of the results.

1 Nilsson IM, Astedt B, Hedner U, Berezin D. Intrauterine death and circulating anticoagulant "Antithromboplastin". Acta Med Scand 1975;197:153-9.

2 Howard MA, Firkin BG, Healey DL, Choong SCC. Lupus anticoagulant in women with multiple spontaneous miscarriage. Am J Hematol 1987;26:175-8.

3 Cowchock S, Smith JB, Gocial B. Antibodies to phospholipids and nuclear antigens in patients with repeated abortions. Am J Obstet Gynecol 1986;155:1002-10.

4 Unander AM, Norberg R, Hahn L, Arfors L. Anticardiolipin antibodies and complement in ninety-nine aron with women with habi

5 Hall R, Malia RG. Medical Laboratory Haematology London: Butterworths, 1984

6 Thiagarajan P, Pengo V, Shapiro SS. The use of the dilute Russell viper venom time for the diagnosis of lupus anticoagulants. Blood 1986;68:869-74.

7 Harris EN, Gharavi AE, Patel SP, Hughes GRV. Evaluation of the anticardiolipin antibody test: report of an international workshop held 4th April, 1986. Clin Exp Immuno 1987;68:215-22.

8 Harris EN, Chan JKH, Asherson RA, Aber VR, Gharavi AE, Hughes GRV. Thrombosis, recurrent fetal loss, thrombocytopenia: predictive value of $\mathrm{IgG}$ anti-cardiolipin antibodies. Arch Intern Med 1986;146:2153-9.

9 Petri M, Golbus M, Anderson R, Whiting O'Keefe $Q$, Corash L, Hellman D. Antinuclear antibody, lupus anticoagulant and anticardiolipin antibody in women with anticoagulant and anticardiolipin antibody in women with idiopathic

10 Kornberg A, Silber L, Yona R, Kaufman S. Clinica manifestations and laboratory findings in patients with lupus anticoagulants. Eur J Haematol 1989;42:90-5.

11 Walport MJ. Pregnancy and antibodies to antiphospholipids. Ann Rheum Dis 1989;48:795-7.

12 The Lupus Anticoagulant Working Party. Detection of lupus like anticoagulant: current laboratory practice in the United Kingdom. J Clin Pathol 1990;43:73-5. 